\title{
INCIDENCIA DEL OCIO EN EL DESARROLLO DE LA CARRERA EN ALUMNOS DE EDUCACIÓN SECUNDARIA OBLIGATORIA
}

\author{
Magdalena Sáenz de Jubera Ocón \\ Universidad de La Rioja
}

RESUMEN: Este artículo plantea una aproximación hacia la Orientación del Desarrollo de la carrera, que se caracteriza por el sentido evolutivo del trabajo a lo largo de la vida como parte de desarrollo personal, que conlleva nuevas perspectivas de intervención orientadora como los procesos de diagnóstico y evaluación de la carrera. Así se realiza un análisis del desarrollo de la carrera en los estudiantes de segundo ciclo de Educación Secundaria Obligatoria, estimando las aficiones de estos jóvenes, como dimensiones del individuo que exige valorar el Diagnóstico para el Desarrollo de la Carrera, a partir de instrumentos adecuados al contexto en el que se desarrolla la madurez vocacional del alumno y analizar su grado de relación con las distintas áreas ocupacionales conforme a las últimas formulaciones del modelo RIASEC de Holland.

Los resultados de diversos análisis estadísticos -sobre una muestra final de 370 alumnos de Educación Secundaria-, indican que los instrumentos utilizados poseen características técnicas adecuadas, que en la planificación del proyecto vocacional tienen una gran influencia las aficiones del alumno y ha permitido establecer relaciones interesantes entre las preferencias profesionales de los estudiantes de Educación Secundaria y las actividades de ocio.

PALABRAS CLAVE: Desarrollo de la carrera, actividades de ocio, áreas ocupacionales, Holland.

\section{IMPACT OF LEISURE ON CAREER DEVELOPMENT OF SECONDARY SCHOOL STUDENTS}

\footnotetext{
ABSTRACT: The purpose of this article is to present an approach to Guidance for Career Development, characterized by the evolutionary sense of the work throughout life as part of personal development, presenting new perspectives on counseling intervention, such as the processes of diagnosis and career
} 
evaluation. So an analysis of career development of students in second cycle of Secondary school is done, considering the leisure activities of these young people as individual dimensions, which requires, on the one hand, to assess the Diagnosis for Career Development, by using instruments appropriate to the context in which vocational maturity of students is developed, and, on the other hand, to analyze its degree of relationship with the different occupational areas according to the latest formulation of Holland s RIASEC Theory.

The results of various statistical analyses -on a final sample of 370 Secondary School students- indicate that the instruments used have appropriate technical characteristics and that students likes have a big influence on the vocational project planning. As well as this, these results have allowed to establish interesting relationships among professional preferences of Secondary School students and their leisure activities.

KEYWORDS: Career development, leisure activities, occupational areas, Holland.

Recibido: 21/10/2011

Aceptado: 29/03/2012

\section{INTRODUCCIÓN}

En la actualidad la dificultad en el acceso al mundo del trabajo, el carácter temporal del mismo, la precariedad de las condiciones laborales, la vulnerabilidad ante el empleo y la dificultad de mantener una trayectoria profesional estable junto con las exigencias profesionales que solicitan nuevas competencias que precisan de la formación para el empleo, las relaciones laborales y la competitividad que se genera en los centros de trabajo hace que sean necesarias personas que no sólo cuenten con conocimientos técnicos sino con habilidades que les permitan interactuar mejor y resolver de forma eficaz los problemas que surjan. Así, factores como las prácticas profesionales no laborales, las estancias formativas en el extranjero, las acciones de voluntariado o las actividades que se realizan en el tiempo libre, propias de la educación no formal e informal, establecen una clara diferencia entre aspirantes a un puesto de trabajo que poseen la misma educación formal. Las transformaciones laborales propias del siglo XXI desafían las nociones tradicionales de carrera y de desarrollo profesional y es necesario incitar a las personas a que participen activamente en la gestión de su propia carrera y enseñarles a construirla (Do Céu Teveira y Rodríguez Moreno, 2010).

Ante esta perspectiva la Orientación para el Desarrollo de la Carrera, así se entiende en la actualidad la Orientación Profesional, como un proceso de ayuda continuo, evolutivo, sistemático, con carácter técnico, preventivo, de desarrollo e intervención social, para lograr el desarrollo de un estilo vital propio a partir de la autocomprensión, la conciencia del propio desarrollo profesional, el significado personal del trabajo, la interrelación entre roles vitales, la adquisición de competencias y el aprendizaje del proceso de toma de decisiones, en último término, la auto-orientación del individuo en su contexto (Mudarra, 2007) se enfrenta a la necesidad de que se den dos con- 
diciones fundamentales, que el individuo se conozca así mismo (factores personales) y comprenda también el entorno sociocultural en el que se desenvuelve (factores profesionales, sociales, contextuales...). En la elección vocacional entran en juego múltiples determinantes, todo depende de la importancia que se otorgue a unos o a otros factores, y a la forma que se relacionan entre sí. El ocio juega un papel sobresaliente en el desarrollo de carrera de los adolescentes porque les da la libertad de explorar, probar, y recibir feedback sobre las direcciones de carrera (Haworth y Smith, 1976; Munson, 1993). Las personas dedicamos gran parte de la jornada al trabajo, del que esperamos adquirir no sólo logros materiales sino también sociales. La actividad profesional es uno de los medios de realización personal. Cuanto más próximo esté el trabajo de las aficiones personales más placentero será para el ser humano y cuanto más fácilmente encuentran salida en todo el campo de sus actividades más éxito tendrá esa persona y más satisfacciones alcanzará.

Por ello, en este trabajo, se consideran estas dimensiones en la elección vocacional y en su desarrollo, puesto que la Orientación para la carrera intenta ofrecer respuestas que hagan posible la mejora de las elecciones por parte de los sujetos, enfatizando la dimensión evolutiva del desarrollo vocacional, al considerar el desarrollo profesional como un proceso que se da a lo largo de toda la vida y que forma parte del desarrollo personal. Desde la Orientación para la carrera se precisa la utilización de instrumentos que hagan posible evaluar estas variables, de modo que sea factible su comprobación empírica. Por ello se utiliza un instrumento diagnóstico que permite conocer las aficiones personales del sujeto con el fin de obtener informaciones que ayuden a los estudiantes en las diferentes elecciones. Este inventario tiene como objetivo hacer reflexionar a los jóvenes sobre una serie de aspectos que le van a conducir a unos campos ocupacionales que guardarán relación con sus aficiones personales, siendo un recurso que puede ayudar al sujeto en su elección ocupacional.

\section{LAS AFICIONES EN LAS ELECCIONES VOCACIONALES}

Tradicionalmente se han tenido más en cuenta los intereses profesionales que las aficiones en las actividades de orientación, siendo un tremendo error, puesto que las aficiones son una importante fuente de satisfacción en la vida del individuo. Las aficiones son necesarias para disfrutar de la vida, son un complemento no indispensable pero si necesario, es importante hacer aquello que a uno le gusta. Por ello, cuando hay conexión entre la afición y la ocupación, produce mayor bienestar en la persona. Desde el proceso de Orientación Profesional hay que dar importancia y estimular las aficiones, dedicarles tiempo y hacer al alumno darse cuenta de la cantidad de actividades agradables para él que puede hacer, el beneficio que obtiene con ellas y la proximidad entre las distintas aficiones y las actividades profesionales.

La actividad de ocio ha sido definida como una actividad intrínsecamente motivada fuera de las actividades de clase (incluidas las actividades informales y las actividades extracurriculares formalmente organizadas) que los estudiantes hacen para su propio placer y por su propia opción, y no por exigencias de clase o por obtener una calificación (Hong, Milgran y Whiston, 1993). 
Numerosos estudios afirman que la práctica de actividades de ocio tiene una influencia positiva y necesaria en el ser humano, y que proporcionan diferentes beneficios. Así, las ventajas psicológicas de experiencias de ocio han sido apoyadas por científicos desde los años cincuenta, ventajas de tipo emocional, cognitivo o conductual. Además los efectos beneficiosos del ocio influyen positivamente en la salud mental y en la condición física, incidiendo en la mejora de la salud y la forma física, e incrementando las habilidades sociales y las relaciones personales. En este sentido la mayor parte de investigadores que estudian las experiencias de ocio afirman que proporcionan al individuo el medio para alcanzar los objetivos sociales, creando situaciones importantes y entornos para el desarrollo social y permitiendo a los que participan de ellas adquirir habilidades útiles, construir una identidad psicosocial, y formular aspiraciones futuras (Glancy, Willits y Farrell, 1986). Finalmente, como señala Cuenca (2009), se hablan de los beneficios globales de las experiencias de ocio, puesto que tienen unos efectos que inciden globalmente en la personalidad, siendo oportunidades de autonomía e independencia, espacios para desarrollar identidades que complementan la función del entorno.

Tinsley, Hinson, Tinsley y Holt (1993) sugirieron que estas ventajas eran resultado de la experiencia fenomenológica del individuo en el ocio más que de la actividad en sí misma. En otras palabras, la experiencia de ocio de una persona incluyendo la cognición (en el sentido de concentración, reto y control) y los afectos (los sentimientos de libertad, el placer y la capacidad) puede darse en varias actividades diarias en el ámbito familiar y en el trabajo (Kelly y Kelly, 1994). Las experiencias de ocio se asocian con los niveles más altos de afectos positivos y se ha demostrado que el ocio requiere de persistencia, esfuerzo y compromiso, características a menudo asociadas con actividades extrínsecamente motivadas o con el trabajo (Tinsley y otros, 1993).

El ocio ocupa una parte considerable de la vida de los estudiantes y estos, además, le otorgan una posición importante en la jerarquía de las actividades que para ellos son significativas. Un porcentaje elevado de tiempo dedicado al ocio se aplica a las actividades que ocurren dentro y fuera del contexto de la escuela: la familia, la comunidad, diversas asociaciones, que enmarcan u organizan con eficacia una parte importante del tiempo libre de los estudiantes. Los centros educativos tienen en su programa un mínimo de contenidos de este tipo, y están generalmente bien dotados de equipamiento deportivo y de talleres de producción sociocultural, por lo tanto, deben contribuir a estructurar el tiempo dedicado al ocio. La práctica de estas actividades ponen en contacto al estudiante con ambientes físicos y de relaciones personales que forman su manera de pensar, hacer y sobre todo de ser, encontrando continuidad con aspectos particulares de la educación, como la exigencia de esfuerzos intelectuales (por ejemplo, resolviendo sudokus, componiendo música, realizando experimentos científicos). Así, se entiende que desde los centros educativos se debe establecer una acción coordinada entre la Orientación y la Educación del Ocio, cuyos objetivos sean el perfeccionamiento, la mejora y la satisfacción vital de los educandos. Como señala Cuenca $(1999,2002)$ el ocio se convierte en ámbito educativo en el momento que se considera como desarrollo humano, como vivencia que contribuye a mejorar la realidad de la persona o de la comunidad, mejoras como conseguir más libertad, ser más autónomos, solidarios, toleran- 
tes, experimentando el ocio desde una vertiente positiva que genera energías que lo convierten en fuente de mejora y por ende, en ámbito educativo.

Una razón de por qué estas actividades realmente pueden predecir el logro adulto es porque su rendimiento requiere no sólo de las capacidades intelectuales anteriormente mencionadas, sino también el compromiso de tarea, la persistencia, y otros atributos cognoscitivos, personales y sociales que determinan en gran medida los resultados que se obtienen en la vida. El aprender a ser y aprender a vivir juntos ganan terreno al conocer y al aprender a hacer en una sociedad con más tiempo libre y en la que el trabajo ocupa menos tiempo (Tractenberg, Streumer y Van Zolingen, 2003, citado en Iriarte, 2004). Parte del aprendizaje informal y la exploración tienen lugar en las actividades de ocio, contribuyendo así a las elecciones ocupacionales. Al respecto, Super (1984) señaló que las actividades de ocio pueden ser experiencias exploratorias valiosas, que proporcionan la oportunidad de experimentar actividades relacionadas ocupacionalmente con ellas, y acentuó la importancia de las aficiones, que tienen su mayor salida en la niñez y la adolescencia, como preparatorias para el papel que el trabajador asume en la juventud y en la adolescencia. Asimismo, Holland (1985) reconoció que las actividades de ocio son escogidas sobre la base de los mismos factores de personalidad y los intereses que dirigen a la persona a la selección de la ocupación.

Tras la aparición del papel de vida y de las perspectivas multiculturales sobre la carrera, las relaciones entre las variables de ocio y los dominios de desarrollo de carrera han sido una cuestión teórica importante. Aunque Super ya avanzara hace más de cincuenta años que el ocio y la carrera influyen mutuamente el uno en el otro, señalando que la participación en el ocio amplía la perspectiva del individuo sobre el mundo del trabajo, porque esto revela opciones diversas, desarrolla habilidades, clarifica valores, aumenta la autoestima, y crea actitudes realistas hacia el trabajo (Glancy y otros, 1986; Holland y Andre, 1987). En particular, Super (1957) afirmó que la participación en las actividades de ocio facilita la síntesis de los procesos de desarrollo profesional. Por ejemplo, las experiencias de ocio pueden formar las elecciones de carrera de los escolares, quienes utilizan las ocasiones de entretenimiento y el role playing para probar el realismo de sus identificaciones ocupacionales.

En el presente trabajo se presentan las aficiones de los jóvenes de Educación Secundaria Obligatoria, como dimensiones del individuo que exige valorar el Diagnóstico para el Desarrollo de la Carrera, a partir de instrumentos adecuados al contexto en el que se desarrolla la madurez vocacional del alumno y se analiza su grado de relación con las distintas áreas ocupacionales conforme a las últimas formulaciones del modelo RIASEC de Holland. En concreto las cuestiones de investigación que se plantean contrastar son: 1) El grado de relación entre las aficiones personales y las áreas ocupacionales en los alumnos riojanos de segundo ciclo de ESO. 2) Las diferencias en función del género, nivel académico y ambiente de centro en las aficiones personales obtenidas por los alumnos objeto de estudio. 3) Las diferencias también en función del género, etapa educativa y ambiente de centro entre los resultados conseguidos en la elección de las áreas ocupacionales, y por último comprobar las prioridades en cuanto a las aficiones y a la elección de las distintas áreas ocupacionales en los alumnos riojanos de $3^{\circ}$ y $4^{\circ}$ de ESO. 


\section{MÉTODO}

El estudio empírico incluye diversas fases y procedimientos metodológicos ajustándose a un diseño de carácter descriptivo o exploratorio.

\section{Muestra}

El estudio se realizó sobre una muestra inicial intencional de 370 alumnos de $3^{\text {o }}$ y $4^{\circ}$ de Educación Secundaria Obligatoria -por ser un nivel crítico respecto a la toma de decisiones vocacionales- asistentes a centros públicos y privados de la Comunidad Autónoma de La Rioja, con edades comprendidas entre 14 y 18 años.

Cómo se puede apreciar en el gráfico 1 la distribución de la muestra por niveles académicos es equitativa, por sexo existe ligero predominio de las mujeres y es equilibrada respecto a la variable ambiente de centro, porcentajes que reflejan muy bien la realidad de la provincia, donde más de la mitad de los habitantes de la Comunidad Autónoma de La Rioja se concentran en la capital y el resto se reparten en pueblos pequeños de la geografía riojana.
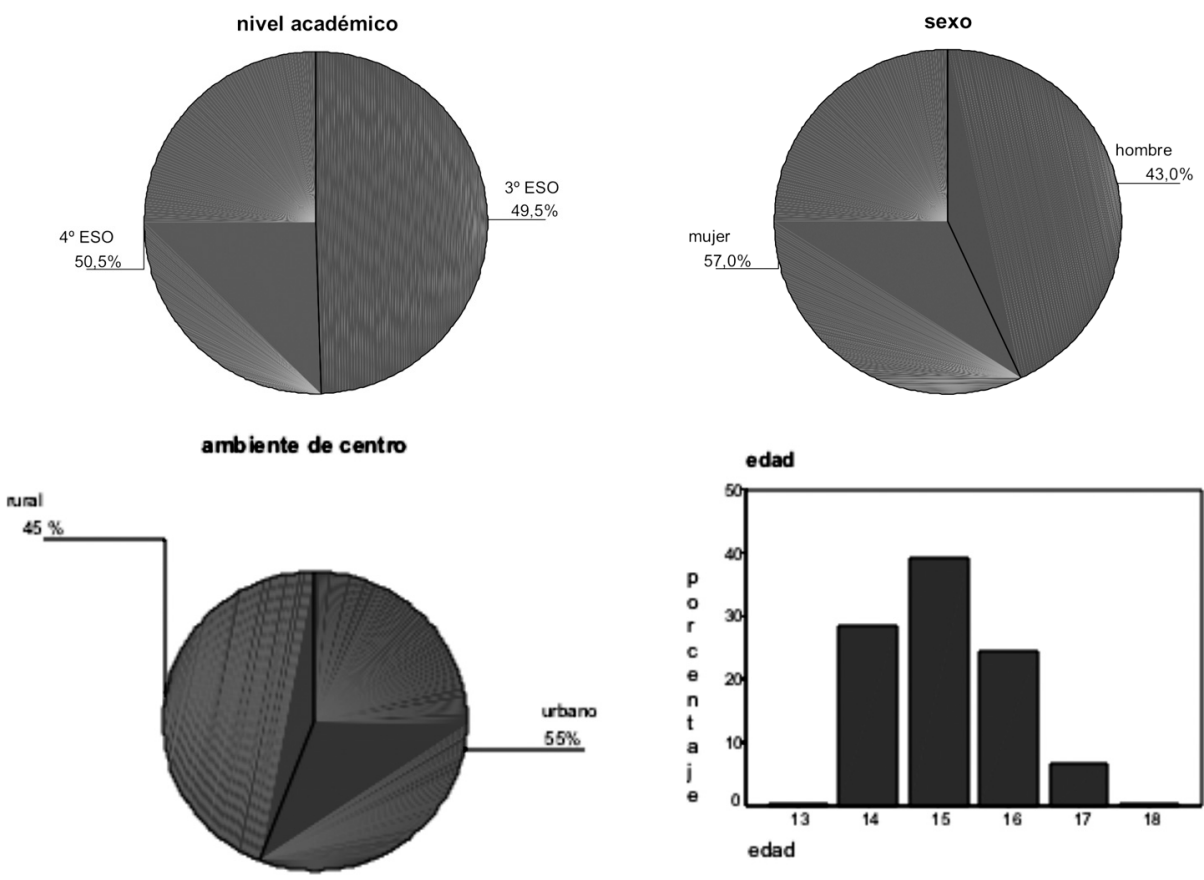

Figura 1. Datos de la muestra

En cuanto a la distribución por edad vemos que la mayoría del alumnado tiene edades comprendidas entre 14 y 16 años (92,4\%), es decir, siguen el curso que corresponden a su edad, supone un porcentaje pequeño los alumnos/as que han repetido algún curso. Sin embargo, si cruzamos las variables edad y curso obtenemos 
unos resultados diferentes, concretamente los que repiten $3^{\circ}$ son el $4,4 \%$, y los que repiten $4^{\circ}$ son el $13,9 \%$, porcentaje muy elevado frente a los alumnos de $3^{\circ}$ de ESO.

\section{Instrumentos aplicados}

Se utiliza el instrumento diagnóstico Cuestionario de Aficiones Personales (Sáenz de Jubera, 2011) para evaluar globalmente las aficiones, cuya información junto a otros elementos del instrumento, también incluye un cuestionario de valores personales, contribuye a facilitar la toma de decisiones vocacionales durante el proceso de Orientación de la Carrera. Se plantean cuestiones referentes a las aficiones personales del sujeto. Este cuestionario está formado por 54 preguntas, seis de las cuales se relacionan con aficiones próximas a las actividades que se llevan a cabo en un campo profesional determinado.

Tratamos de poner de manifiesto las preferencias cuantificadas de 1 a 5 puntos de las aficiones personales presentadas en las distintas cuestiones al sujeto, y que el alumno/a tenga conciencia de sus aficiones y estime la importancia de tenerlas en cuenta en la toma de decisiones.

Se incluyen cuestiones que hacen referencia a las aficiones siguientes:

- Aficiones humanístico-asistenciales: Preferencia por actividades de trato con los demás para prestarles algún tipo de ayuda o colaborar con ellas.

- Aficiones económico-empresariales: Preferencia por actividades de ventas, contables, negocios y empresas.

- Aficiones de carácter biológico: Preferencia por actividades ligadas a la naturaleza y a los seres vivos.

- Aficiones al aire libre-deportes: Preferencia por actividades al aire libre y relacionadas con el mundo del deporte.

- Aficiones de carácter teórico: Preferencia por actividades intelectuales, de descubrimiento de nuevos hechos y resolución de problemas.

- Aficiones de carácter técnico-práctico: Preferencia por actividades técnicas y mecánicas.

- Aficiones de carácter socio-político: Preferencia por actividades sociales y de carácter político.

- Aficiones de carácter religioso-trascendental: Preferencia por actividades relacionadas con lo trascendental y lo religioso.

- Aficiones de carácter artístico: Preferencia por actividades manuales creativas.

Como resultado se obtiene un perfil que identifica las aficiones de la persona y el orden de prioridad de éstas.

En cuanto a la fiabilidad del instrumento procedimos a calcularla mediante el coeficiente alfa de Cronbach, cruzando todas las variables entre sí. El coeficiente del instrumento es de 0.8901 en la escala de aficiones, obteniendo índices de fiabilidad adecuados conforme los que se suelen encontrar en los instrumentos de diagnóstico de intereses y personalidad, en torno al 0.8 (Pérez Juste, 1985).

Respecto a los análisis factoriales se confirmó la estructura de 9 componentes, definidos fundamentalmente por los ítems correspondientes a cada afición. No obstante, se precisó la definición de tres ítems que saturaban significativamente y con valores muy próximos en los factores. 
El otro instrumento empleado es La Llave de la Carrera de Jones, 1989 (versión en español de Lázaro, A.; Mudarra, M.J. y Casarrubias, R.). Los estilos vitales o tipos del modelo RIASEC de Holland es su referente teórico. Es un instrumento que fue diseñado para incrementar en sus usuarios la adquisición de una estructura cognitiva adecuada que favoreciera su desarrollo de carrera y mejorase su nivel de información ocupacional, lo que se suponía tendría un efecto positivo en dicho desarrollo. La Llave de la Carrera es un cuadernillo auto-aplicado que se estructura en 5 secciones, en las 3 primeras sus destinatarios se autoevalúan conforme los seis tipos de personalidad de Holland: Realista, Investigador, Artístico, Social, Emprendedor y Convencional (RIASEC) valorando en la primera el grado de verdad de una serie de afirmaciones descriptivas de la persona, estimando en la segunda el grado de interés del usuario en cada una de las ocupaciones que se presentan agrupadas conforme a los seis tipos de Holland y llevando a cabo en la tercera la valoración glotal en cada tipo mediante la suma de las puntuaciones totales de las dos secciones. En la cuarta sección el usuario puede identificar nuevas ocupaciones al consultar una lista de ocupaciones organizada de acuerdo a dichos tipos y en la quinta sección se ofrecen algunos pasos a realizar para continuar con la exploración de la carrera y facilitar la toma de decisiones.

En cuanto a las características técnicas la Llave de la Carrera en muestras norteamericanas la fiabilidad medida con el coeficiente alfa de Cronbach es de 0.8014, una fiabilidad alta. La validación española de la Llave de la Carrera realizada por Lázaro, A. y Mudarra, M.J. en Lázaro, A. (2000), obtiene índices de fiabilidad incluso superiores en algunas escalas, a las obtenidas por Jones. Respecto a la Validez criterial, valoraron el grado de validez de cada sub-escala o tipo de la Llave de la Carrera, es decir, partieron de la hipótesis de que debe existir seis dimensiones o tipos subyacentes al constructo de vocación expuesto por Holland, lo que puede determinarse mediante las correlaciones entre cada tipo y su tipo análogo tal como es evaluado en otro instrumento paralelo, en este caso, la Investigación Autodirigida de Holland. Todas las correlaciones son significativas a un nivel de 0.01 y la validez en las diversas subescalas es bastante alta, por tanto la dimensionalidad de la Llave de la Carrera se confirma, produciéndose la mayor coincidencia con los tipos de Investigación Autodirigida en el caso de la sub-escala artística y de la investigadora.

Así, para este estudio, se ha utilizado la $2^{\text {a }}$ sección del instrumento denominada OCUPACIONES para la obtención de puntuaciones correspondientes a la medición de las áreas ocupacionales propuestas por Holland:

- Realista: Ocupaciones manejo de instrumentos o máquinas.

- Investigadora: Ocupaciones de tipo científico.

- Artística: Ocupaciones de tipo artístico.

- Social: Ocupaciones en las que se tenga que participar socialmente.

- Emprendedora: Ocupaciones en las que se desempeña esa actitud.

- Convencional: Ocupaciones tradicionales, valora aspectos económicos.

En esta sección, se presentan 36 ocupaciones extraídas del Diccionario de Códigos ocupacionales (DHOC) de Holland agrupadas conforme los seis tipos de Holland, cada una de ellas supone un ítem, es decir, hay seis ítems para cada tipo, para que estime el grado de interés que despiertan en él cada una de ellas, el cual puede oscilar entre 0,1 y 2 . 


\section{Procedimiento}

La primera aplicación de las pruebas permitió valorar las necesidades de orientación para el desarrollo de la carrera de los alumnos considerados, siendo el punto de partida para una versión definitiva de la prueba diagnóstica dirigida a la población escolar de la Comunidad Autónoma de La Rioja. Una vez elaborado éste y determinada la muestra representativa de la población, se comenzó a aplicar a los centros seleccionados. La aplicación de los cuestionarios al alumnado de la muestra tuvo lugar durante los horarios normales de clase. Se respetó siempre el orden de aplicación, primero el cuestionario de aficiones personales y después la Llave de la Carrera de Jones. Para analizar las características técnicas de los instrumentos y comprobar las cuestiones de investigación planteadas, se utilizaron diversos procedimientos de análisis estadísticos con el programa SPSS, como índices de validez, análisis factoriales de componentes principales, análisis correlacionales y análisis multivariados de varianza.

\section{DisCusión DE RESULTADOS}

A continuación se presentan los principales resultados de los análisis efectuados.

La primera cuestión que nos planteamos es averiguar cuáles son las prioridades en la elección de las áreas ocupacionales y aficiones personales. Para ello se llevará a cabo un análisis de los porcentajes de valoración.

Respecto a las aficiones personales, se aprecia -ver cuadro 1- que sobre un valor máximo de 30, destacan las bajas puntuaciones obtenidas en las aficiones religiosotrascendentales (IX) seguidas muy de cerca por las aficiones biológicas (IV), si bien en estas últimas los alumnos/as de $3^{\circ}$ y $4^{\circ}$ de ESO puntúan por encima de la media. Esto puede ser explicable por los momentos actuales de crisis de muchos principios de carácter ético religioso o moral. A bastante distancia del resto de aficiones, las puntuaciones más altas se sitúan en las aficiones deportivas (VI), lo que nos indica que los estudiantes cada día dedican más su tiempo libre a la práctica deportiva y a ver deporte tanto en los campos de juego como a través de las tecnologías de información y comunicación.

\section{Aficiones}

6. Deportivas 23,3405 (77,80\%)

7. Sociopolíticas $19,1541(63.84)$

3. Artísticas $18,9405(63.13 \%)$

5. Humanísticas $18,9495(63.13 \%)$

1. Técnico Pr. 18,0216 (60.07\%)

2. Intelectuales 17,7324 (59.11\%)

8. Administrativo Ec. 16,3459 (54.49\%)

4. Biológicas $15,4946(51.65 \%)$

9. Religioso T. $14,2676(47.56 \%)$
}

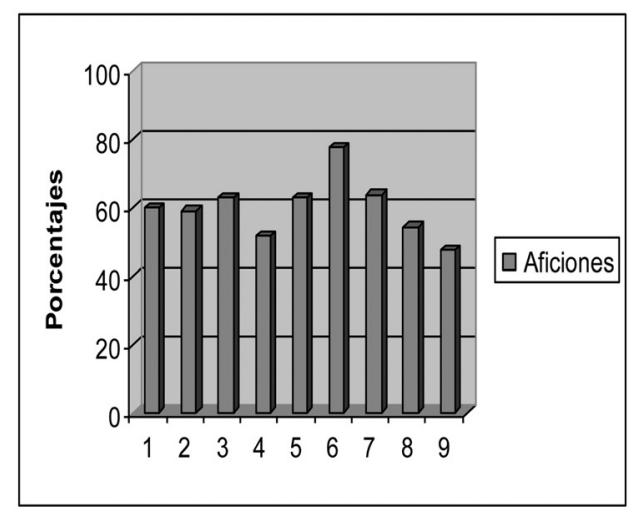

Figura 2. Distribución porcentual de las Aficiones Personales 
En cuanto a las elecciones ocupacionales destaca -ver cuadro 2- que sobre un valor máximo de 12, sobresalen las bajas puntuaciones obtenidas en todas las áreas, siendo el área Convencional a la que los alumnos/as de $3^{\circ}$ y $4^{\circ}$ de ESO conceden menor puntuación. Por otro lado, las puntuaciones más altas se sitúan en el área artística, lo que nos indica que los estudiantes consideran atractivas ocupaciones que tienen mucho que ver con el mundo del espectáculo: actores, cantantes, músicos... Por detrás de éstas se encuentran las ocupaciones sociales, relacionadas con las profesiones de corte humanístico-asistencial y de ayuda a los demás.

\section{Áreas ocupacionales \\ 口 $1^{\circ}$ Área Artística, 4.74 \\ $\square \quad 2^{\circ}$ Área Social, 4.03 \\ 口 $3^{\circ}$ Área Intelectual, 3.73 \\ 口 $4^{\circ}$ Área Emprendedora, 3.02 \\ 口 $5^{\circ}$ Área Realista 2.90 \\ 口 $6{ }^{\circ}$ Área Convencional. 2.67}

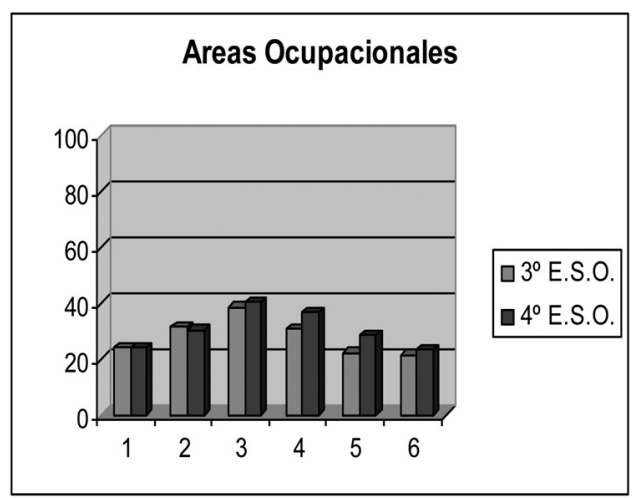

Figura 3. Distribución porcentual por cursos de las Áreas Ocupacionales

La segunda cuestión que nos ocupa es comprobar si es significativo el grado de relación entre las aficiones personales y las áreas ocupacionales. Para dar respuesta a esta cuestión se calcula el coeficiente de correlación lineal y se somete este coeficiente a un test de significación estadística para evidenciar la relación estimativa entre las áreas ocupacionales y las aficiones personales.

Observando la matriz que presenta la correlación existente entre las aficiones personales (Cuadro 3), podemos apreciar que:

El Área Realista obtiene un índice de correlación moderado con las Aficiones Técnico Prácticas $(0,449)$.

El Área Investigadora correlaciona positivamente con las Aficiones Intelectuales $(0,476)$ y las Aficiones Biológicas $(0,407)$.

Con respecto al Área Artística, el nivel de correlación es el mismo y es significativamente moderado con las aficiones Artísticas $(0,459)$ y Humanístico-Asistenciales $(0,459)$.

El Área Social correlaciona positivamente con las Aficiones Humanístico-Asistenciales $(0,567)$ y las aficiones Artísticas $(0,567)$. El valor del índice de contingencia es idéntico.

El Área Emprendedora obtiene un índice de correlación moderado con las Aficiones Administrativo-Económicas $(0,435)$ y Socio-políticas $(0,373)$.

El Área Convencional se correlaciona con las Aficiones Administrativo-Económicas $(0,435)$. 
Respecto a la tercera cuestión, si existen diferencias significativas en función del género, nivel académico y lugar de procedencia en las aficiones, se plantea un análisis de varianza entre cada una de las aficiones clasificadas en función del sexo, nivel académico y ubicación del centro educativo: rural/urbana. Para aplicar este análisis de varianza es preciso que se cumplan los supuestos de aleatoriedad, normalidad y homocedasticidad. Para comprobar la igualdad de varianzas se ha utilizado el test de Levenne.

De los resultados obtenidos en el análisis de varianza (Cuadro 4) podemos extraer las siguientes conclusiones:

A un nivel de significatividad del 1\%, existen diferencias en relación al género en los resultados obtenidos en las siguientes aficiones: Técnico-Prácticas, presentan mayor interés los varones que las mujeres (20,09 de media para ellos y 16,45 para ellas); Artísticas, son las mujeres las que tienen mayor predilección (20,86 para las alumnas y 16,38 para los alumnos); Humanístico-Asistenciales, presentan mayor interés las chicas que los chicos (20,09 de media para ellas y 16,45 para ellos) y Sociopolíticas, asignan mayor valor las mujeres que los hombres (19,80 para ellas y 18,29 para ellos).

No existe diferencia en función del sexo en cuanto al valor que concede el alumnado a las Aficiones Intelectuales, Biológicas, Deportivas, Administrativo-económica y Religioso-trascendentales.

Por otra parte, no existen diferencias significativas a un nivel del $1 \%$ en relación al nivel académico ni al ambiente de centro sobre todas las Aficiones personales estudiadas.

En la cuarta cuestión, relativa a si hay diferencias significativas en función del género, etapa educativa y ambiente de centro en la elección de las áreas ocupacionales, se presenta el mismo problema que en la cuestión anterior, pero ahora para las áreas ocupacionales. Se realiza un análisis de varianza entre cada una de las áreas ocupacionales analizadas en función del sexo, nivel académico y ambiente de centro.

Tras el análisis de varianza (Cuadro 5) concluimos que:

Existen diferencias significativas a un nivel del $1 \%$ en relación al sexo en las valoraciones que hacen los estudiantes de primer ciclo de ESO sobre las áreas ocupacionales Realista, presentan mayor interés los varones que las mujeres $(4,78$ de media para ellos y 1,4653 para ellas); Artística, asignan mayor valor las mujeres que los hombres (5,30 para ellas y 4,00 para ellos) y Social, presentan mayor interés las chicas que los chicos (4,51 de media para ellas y 3,39 para ellos). Se observa que existe diferencia en favor de los hombres en el área Realista, mientras que las mujeres muestran preferencia por las Áreas Artística y Social.

No existen diferencias significativas en las áreas Intelectual, Emprendedora y Convencional.

Al igual que en las aficiones no existen diferencias significativas a un nivel del $1 \%$ en función de las variables ambiente de centro y nivel académico en cuanto a las valoraciones que conceden los alumnos/as a las Áreas ocupacionales estudiadas.

\section{Conclusiones}

Respecto a la determinación de las variables que intervienen en los procesos de exploración de la carrera concluimos que el desarrollo de la madurez de carrera es 
un aspecto cardinal en la vida de los individuos y las decisiones que tomen los jóvenes en este ámbito tienen importantes implicaciones personales, sociales y económicas. En la planificación del proyecto vocacional tienen una gran influencia, entre otras variables, las aficiones del sujeto. Resulta que las aficiones personales y las áreas ocupacionales están moderadamente relacionadas, lo que nos indica que el ocio puede ser un predictor válido de las elecciones ocupacionales, de ahí, la importancia de utilizar un instrumento diagnóstico para el desarrollo de Carrera que evalúe las características de las aficiones personales en los alumnos de Educación Secundaria Obligatoria.

Además, se han establecido algunas relaciones interesantes entre las preferencias profesionales de los estudiantes de $3^{\circ}$ y $4^{\circ}$ de Educación Secundaria y las actividades de ocio. Concretamente, se ha demostrado que existe una relación significativa entre las aficiones Técnico-prácticas ejercidas por estos sujetos y las ocupaciones de tipo Realista, entre las aficiones Intelectuales y Biológicas y las profesiones del sector Investigador; entre las Aficiones Humanístico-Asistenciales y Artísticas y los trabajos de tipo Social; entre las Aficiones Administrativo económicas y Sociopolíticas y las ocupaciones pertenecientes al Área Emprendedora; entre las actividades de ocio Administrativo-Económicas y las profesiones de tipo Convencional y por último, existe también una relación sólida entre las aficiones Artísticas y HumanísticoAsistenciales y el Área ocupacional Artística. Un índice de correlación bajo, aunque significativo obtienen las Aficiones Deportivas y Biológicas con el área Realista; las Técnicas, Sociopolíticas y Religioso-Trascendentales con el Área Intelectual; las Humanístico-Asistenciales, Intelectuales y Sociopolíticas con el Área Artística; las Intelectuales y Deportivas, Sociopolíticas y Artísticas con el Área Social, las Deportivas y Técnicas con el Área Emprendedora y las Sociopolíticas con el Área Convencional.

En el campo de la orientación, estos resultados llevan a creer que sería deseable que los alumnos tengan la posibilidad de aprender a conocerse, utilizar la exploración de sus actividades de ocio para ayudarles a aumentar su conocimiento de sí mismos. Estimular y fomentar la capacidad reflexiva de los alumnos en torno las aficiones para que sean ellos mismos los que establezcan su propia escala y orienten sus acciones en consonancia con las preferencias por ellos establecidas. Entendiendo que los estudiantes a través de su itinerario educativo han ido conformándola y que influirán en sus decisiones vocacionales.

Sin embargo, esto debería ser complementado con otros métodos, porque este estudio ha permitido observar que la gente joven tiende a limitarse sólo a los empleos que conocen, por ello los orientadores deberían conceder importancia a extrapolar este conocimiento hacia empleos menos conocidos por los estudiantes. De un lado más científico, esta investigación ha permitido observar como en otros estudios efectuados que existe cierta relación entre las actividades de ocio y las preferencias profesionales, pero que esta relación es moderada.

En referencia a la tipología de Holland, las áreas ocupacionales para el conjunto de los alumnos de $3^{\circ}$ y $4^{\circ}$ de ESO de la Comunidad Autónoma de La Rioja presentan la siguiente configuración: ARTÍSTICA, SOCIAL, INTELECTUAL, EMPRENDEDORA, REALISTA y CONVENCIONAL (ASIERC). Tanto los alumnos de $3^{\circ}$ como los de $4^{\circ}$ obtienen esta misma jerarquía ocupacional, sin embargo los estudiantes de $4^{\circ}$ han obte- 
nido puntuaciones más elevadas que los alumnos de 3 o․ Si tenemos en cuenta la distribución de tipos sobre el hexágono de Holland, un perfil será consistente si los tipos que lo forman son adyacentes en el hexágono, calculados a partir de los dos primeros tipos (AS) obtendríamos en este caso una consistencia alta, lo que sería un indicador de madurez vocacional y estabilidad en la elección vocacional. Si tenemos en cuenta además, que los grados de consistencia influyen sobre las preferencias vocacionales y dado que el grado de consistencia es alto, también lo serán las probabilidades de acierto al predecirlas.

El mayor porcentaje de alumnos/as según el código de HOLLAND está dominado por las áreas Artística y Social. Si bien, estos chicos y chicas poseen intereses ocupacionales diferentes. En nuestro estudio hemos obtenido los perfiles de tipo RAIESC para los chicos y para las chicas ASIECR.

Examinando la jerarquía de las profesiones de los chicos y las chicas, constatamos que lo que prevalece para el conjunto de los sujetos objeto de estudio refleja la realidad de los chicos y chicas de estos niveles educativos. Estos últimos muestran mucho interés por ocupaciones de tipo Realista y menor predilección por actividades donde las relaciones interpersonales son frecuentes. Los intereses de las alumnas son diferentes, puesto que ellas han obtenido puntuaciones más elevadas en la mayoría de las áreas. Se advierten preferencias por las escalas de tipo Artístico, Social e Intelectual y un cierto desagrado por las ocupaciones de tipo Realista. En estas elecciones se nota una marcada preferencia por actividades profesionales donde la creatividad, el contacto humano y la formación académica están implícitos y los intereses por profesiones tradicionalmente "femeninas".

Las mayores diferencias entre las chicas y los chicos de estos niveles educativos se encuentran en las escalas REALISTA y SOCIAL. Teóricamente, esto se traduce en una distancia psicológica importante (Holland, 1973).

\section{REFERENCIAS BIBLIOGRÁFICAS}

CUENCA CABEZA, M. (1999). Ocio y orientación: un encuentro necesario. Revista de ciencias de la educación, 177, 35-50.

CUENCA CABEZA, M. (2002). La educación del ocio: ámbitos de acción futura. Revista de educación, $n^{\circ}$ extra 1, 149-168.

CUENCA CABEZA, M. (2009). Perspectivas actuales de la pedagogía del ocio y el tiempo libre. En Otero López, J. (coord.). La pedagogía del ocio: nuevos desafíos. Lugo: Axac.

DO CÉU TAVEIRA, M. y RODRÍGUEZ MORENO, M. L. (2010). La gestión personal de la carrera y el papel de la orientación profesional. Revista Española de Orientación y Psicopedagogía, 21, 335-345.

GLANCY, M.; WILLITS, F. K. y FARRELL, P. (1986). Adolescent activities and adult success and happiness: Twenty four years later. Sociology and Social Research, 70, 242-247.

HAWORTH, J. T. y SMITH, M. A. (1976). Work and leisure. Princeton, NJ: Princeton Book. 
HOLLAND, J. L. (1973). Making vocational choices: a theory of careers. Englewood Cliffs, NJ: Prentice Hall.

HOLLAND, J. L. (1985). Making Vocational choices: A theory of vocational personalities and work environments ( $2^{\text {nd }}$ ed.). Englewood Cliffs, NJ: Prentice Hall.

HOLLAND, A. y ANDRE, T. (1987). Participation in extracurricular activities in secondary school: What is known, what needs to be known? Review of Educational Research, 57, 437-466.

HONG, E.; MILGRAM, R. M. y WHISTON, S. C. (1993). Leisure activities in adolescents as a predictor of occupational choice in young adults: A longitudinal study. Jounal of career Development, 19, 221-229.

IRIARTE REDÍN, C. (2004). Orientar para la vida a través de la orientación para la carrera. Estudios sobre Educación, 7, 21-32.

JONES, L. K. (1987). The Career Key. Chicago: J.G. Ferguson.

JONES, L. K.; GORMAN, S. y SCHROEDER, C. G. (1989). A comparison between the SDS and the Career Key among Career Undecide College Students. The Career Development Quartely, 37, 334-344.

KELLY, J. R., y KELLY, J. R. (1994). Multiple dimensions of meaning in the domains of work, family, and leisure. Journal of Leisure Research, 26 (3), 250-274.

LÁZARO, A. (2000). Aproximación al estudio de la madurez vocacional en alumnos de Educación Secundaria (inédito). Madrid: Universidad Complutense de Madrid.

MUDARRA, M. J. (2007). El sistema de autoevaluación de áreas profesionales: un instrumento de diagnóstico y Orientación profesional. Educación XX1, 10, 195-213.

MUNSON, W. W. (1993). Perceived freedom in leisure and career salience in adolescence. Journal of Leisure Research, 25, 305-314.

PÉREZ JUSTE, R. (1985). Investigación ex post-facto. En De la Orden, A. (coord.). Investigación Educativa. Diccionario de CC de la Educación. Madrid: Anaya.

SÁENZ DE JUBERA, M. (2011). Incidencia del Ocio y los valores en el desarrollo de la carrera de alumnos de Educación Secundaria Obligatoria. Tesis doctoral. Madrid: Universidad de Alcalá.

SAVICKAS, M. L. (1998). Relation between Leisure and Career Development of College Students. Journal of Vocational Behavior, 53 (2), 243-253.

SUPER, D. E. (1984). Leisure: What is and might be. Journal of Career Development, 10, 71-79.

SUPER, D. E. (1957). The Psychology of Careers. New York: Harper and Row.

TYNSLEY, H. E.; HINSON, J. A.; TINSLEY, D. J. y HOLT, M. S. (1993). Attributes of leisure and work experiences. Journal of counseling Psychology, 40, 447-455.

VALLS FERNÁNDEZ, F. y MARTÍNEZ VICENTE, J. M. (2001). Validez transcultural del modelo hexagonal de personalidad propuesto en la teoría tipológica de elección vocacional de John Holland. Revista de Psicología General y Aplicada, 54 (4), 577-586. 


\section{ANEXOS}

Cuadro1. Estadísticas de las distintas aficiones personales

\begin{tabular}{|c|c|c|c|c|c|c|c|c|c|}
\hline & I & II & III & IV & V & VI & VII & VIII & IX \\
\hline Media & 18,0216 & 17,7324 & 18,9405 & 15,4946 & 18,9405 & 23,3405 & 19,1541 & 16,3459 & 14,2676 \\
\hline Desv. & 4,9512 & 5 & 5,1814 & 4,8255 & 4 & 49783 & 4 & ? & 4,1933 \\
\hline Percentiles 25 & 14,7500 & 15,0000 & 15,0000 & 12,0000 & 15,0000 & 20,7500 & 16,0000 & 13,0000 & 11,0000 \\
\hline 50 & 18,0000 & 18,0000 & 19,0000 & 15,0000 & 19,0000 & 24,0000 & 19,0000 & 16,0000 & 14,0000 \\
\hline 75 & 22,0000 & 21,0000 & 23,0000 & 19,0000 & 23,0000 & 27,0000 & 22,0000 & 19,0000 & 17,0000 \\
\hline
\end{tabular}

Cuadro 2. Estadísticas de las distintas Áreas Ocupacionales

\begin{tabular}{|l|c|c|c|c|c|c|}
\hline & Área Realista & Área Intelectual & Área Artística & Área Social & Área Emprendedora & Área Convencional \\
\hline Media & 2,9017 & 3,7303 & 4,7444 & 4,0337 & 3,0281 & 2,6770 \\
\hline Desv. tip. & 3,0003 & 3,1301 & 2,9673 & 2,4285 & 2,5163 & 2,5175 \\
\hline \multirow{2}{*}{$\begin{array}{r}\text { Percentiles } 25 \\
50\end{array}$} & 0,0000 & 1,0000 & 2,0000 & 2,0000 & 1,0000 & 0,2500 \\
\cline { 2 - 7 } & 2,0000 & 3,0000 & 5,0000 & 4,0000 & 3,0000 & 2,0000 \\
\cline { 2 - 7 } & 5,0000 & 6,0000 & 7,0000 & 6,0000 & 4,7500 & 4,0000 \\
\hline
\end{tabular}

Cuadro 3. Valores de los coeficientes de correlación y niveles de significación para las áreas ocupacionales y las aficiones personales

\section{Correlaciones}

\begin{tabular}{|c|c|c|c|c|c|c|c|c|c|c|}
\hline & & $\begin{array}{l}\text { Aficiones } \\
\text { Técnico } \\
\text { prácticas }\end{array}$ & $\begin{array}{c}\text { Aficiones } \\
\text { Intelectuales }\end{array}$ & $\begin{array}{l}\text { Aficiones } \\
\text { Artísticas }\end{array}$ & $\begin{array}{l}\text { Aficiones } \\
\text { Biológicas }\end{array}$ & $\begin{array}{l}\text { Aficiones } \\
\text { Humanístico } \\
\text { Asistenciales }\end{array}$ & $\begin{array}{l}\text { Aficiones } \\
\text { Deportivas } \\
\text { Aire-libre }\end{array}$ & $\begin{array}{c}\text { Aficiones } \\
\text { Sociopolíticas }\end{array}$ & $\begin{array}{l}\text { Aficiones } \\
\text { Adminis. } \\
\text { Económicas }\end{array}$ & $\begin{array}{l}\text { Aficiones } \\
\text { Religioso } \\
\text { Trascendental }\end{array}$ \\
\hline $\begin{array}{l}\text { Área } \\
\text { Realista }\end{array}$ & $\begin{array}{l}\text { Pearson } \\
\text { Sig. (bilateral) }\end{array}$ & $\begin{array}{c}, 449^{* *} \\
, 000\end{array}$ & $\begin{array}{c}-, 117^{*} \\
, 027\end{array}$ & $\begin{array}{c}-, 274^{* *} \\
, 000\end{array}$ & $\begin{array}{c}, 137^{* *} \\
, 010\end{array}$ & $\begin{array}{c}-, 274^{* *} \\
, 000\end{array}$ & $\begin{array}{c}, 139 * * \\
, 009\end{array}$ & $\begin{array}{c}-, 155^{* *} \\
, 003\end{array}$ & $\begin{array}{c}-, 121^{*} \\
, 022\end{array}$ & $\begin{array}{l}-, 019 \\
, 725\end{array}$ \\
\hline $\begin{array}{l}\text { Área } \\
\text { Intelectual }\end{array}$ & $\begin{array}{l}\text { Pearson } \\
\text { Sig. (bilateral) }\end{array}$ & $\begin{array}{c}, 175^{* *} \\
, 001\end{array}$ & $\begin{array}{c}, 476^{* *} \\
, 001\end{array}$ & $\begin{array}{l}, 129^{*} \\
, 014\end{array}$ & $\begin{array}{c}, 407^{* *} \\
, 000\end{array}$ & $\begin{array}{l}129^{*} \\
, 014\end{array}$ & $\begin{array}{l}107^{*} \\
, 043\end{array}$ & $\begin{array}{c}, 144^{* *} \\
, 006\end{array}$ & $\begin{array}{c}, 238^{* *} \\
, 000\end{array}$ & $\begin{array}{l}163^{* *} \\
, 002\end{array}$ \\
\hline $\begin{array}{l}\text { Área } \\
\text { Artística }\end{array}$ & $\begin{array}{l}\text { Pearson } \\
\text { Sig. (bilateral) }\end{array}$ & $\begin{array}{l}, 071 \\
, 181 \\
\end{array}$ & $\begin{array}{c}140^{* *} \\
, 008\end{array}$ & $\begin{array}{c}, 459^{* *} \\
, 003\end{array}$ & $\begin{array}{l}-, 012 \\
, 823\end{array}$ & $\begin{array}{c}459 * * \\
, 003 \\
\end{array}$ & $\begin{array}{l}, 017 \\
, 749\end{array}$ & $\begin{array}{c}, 198^{* *} \\
, 000\end{array}$ & $\begin{array}{l}, 072 \\
, 172 \\
\end{array}$ & $\begin{array}{l}, 094 \\
, 075\end{array}$ \\
\hline $\begin{array}{l}\text { Área } \\
\text { Social }\end{array}$ & $\begin{array}{l}\text { Pearson } \\
\text { Sig. (bilateral) }\end{array}$ & $\begin{array}{l}-, 040 \\
, 449\end{array}$ & $\begin{array}{c}, 151^{* *} \\
, 004\end{array}$ & $\begin{array}{c}, 567^{* *} \\
, 000\end{array}$ & $\begin{array}{l}, 010 \\
, 845\end{array}$ & $\begin{array}{c}, 567^{* *} \\
, 000\end{array}$ & $\begin{array}{c}, 200^{* *} \\
, 000\end{array}$ & $\begin{array}{c}, 304^{* *} \\
, 000\end{array}$ & $\begin{array}{l}, 111^{*} \\
, 036\end{array}$ & $\begin{array}{l}, 113^{*} \\
, 033\end{array}$ \\
\hline $\begin{array}{l}\text { Área } \\
\text { Emprendedora }\end{array}$ & $\begin{array}{l}\text { Pearson } \\
\text { Sig. (bilateral) }\end{array}$ & $\begin{array}{c}, 151^{* *} \\
, 004\end{array}$ & $\begin{array}{l}, 032 \\
, 553\end{array}$ & $\begin{array}{l}, 016 \\
, 760\end{array}$ & $\begin{array}{l}-, 020 \\
, 711\end{array}$ & $\begin{array}{l}, 016 \\
, 760\end{array}$ & $\begin{array}{l}, 125^{*} \\
, 019\end{array}$ & $\begin{array}{c}, 373^{* *} \\
, 001\end{array}$ & $\begin{array}{c}, 413^{* *} \\
, 000\end{array}$ & $\begin{array}{l}, 042 \\
, 434\end{array}$ \\
\hline $\begin{array}{l}\text { Área } \\
\text { Convencional }\end{array}$ & $\begin{array}{l}\text { Pearson } \\
\text { Sig. (bilateral) }\end{array}$ & $\begin{array}{c}, 123^{*} \\
, 021\end{array}$ & $\begin{array}{l}, 113^{*} \\
, 034 \\
\end{array}$ & $\begin{array}{l}-, 033 \\
, 538\end{array}$ & $\begin{array}{l}-, 024 \\
, 649\end{array}$ & $\begin{array}{r}-, 033 \\
, 538 \\
\end{array}$ & $\begin{array}{l}, 067 \\
, 210\end{array}$ & $\begin{array}{c}, 170^{* *} \\
, 001\end{array}$ & $\begin{array}{c}, 435^{* *} \\
, 000\end{array}$ & $\begin{array}{l}, 095 \\
, 074\end{array}$ \\
\hline
\end{tabular}

** La correlación es significativa al nivel 0,01 (bilateral).

* La correlación es significativa al nivel 0,05 (bilateral). 
Cuadro 4. Valores de $F$ y nivel de significación para cada una de las aficiones personales y la variable sexo

ANOVA

\begin{tabular}{|l|r|c|}
\hline & F & Sig. \\
\hline Aficiones Técnico Prácticas & 56,168 &, 000 \\
\hline Aficiones Intelectuales & 1,054 &, 305 \\
\hline Aficiones Artísticas & 82,979 &, 000 \\
\hline Aficiones Biológicas &, 304 &, 582 \\
\hline Aficiones Humanístico-Asistenciales & 82,979 &, 000 \\
\hline Aficiones Deportivas-Aire libre &, 131 &, 718 \\
\hline Aficiones Sociopolíticas & 11,951 &, 001 \\
\hline Aficiones Administrativo-Económicas &, 623 &, 430 \\
\hline Aficiones Religioso-Trascendentales & 2,307 &, 130 \\
\hline
\end{tabular}

Cuadro 5. Resultados del Análisis de varianza para cada una de las áreas ocupacionales y la variable sexo

ANOVA

\begin{tabular}{|l|r|c|}
\hline & F & Sig. \\
\hline Área Realista & 152,780 &, 000 \\
\hline Área Intelectual &, 281 &, 596 \\
\hline Área Artística & 17,566 &, 000 \\
\hline Área Social & 19,695 &, 000 \\
\hline Área Emprendedora & 6,762 &, 010 \\
\hline Área Convencional & 2,448 &, 119 \\
\hline
\end{tabular}

